\section{Prevalência de sedentarismo nas mulheres adultas da cidade de São Leopoldo, Rio Grande do Sul, Brasil}

\author{
Prevalence of physical inactivity in adult women \\ in São Leopoldo, Rio Grande do Sul, Brazil
}

Carmen Rosane Masson 1

Juvenal Soares Dias-da-Costa 1,2

Maria Teresa Anselmo Olinto 1

Stella Meneghel 1

Clarice Cardoso da Costa ${ }^{1}$

Fernanda Bairros 1

Pedro Curi Hallal 1,2
1 Programa de Pós-graduação em Saúde Coletiva, Universidade do Vale do Rio dos Sinos, São Leopoldo, Brasil.

2 Faculdade de Medicina,

Universidade Federal

de Pelotas, Pelotas, Brasil.

Correspondência J. S. Dias-da-Costa

Departamento de Medicina

Social, Faculdade de

Medicina, Universidade

Federal de Pelotas.

Av. Duque de Caxias 250

3o piso, Pelotas, $R S$

96030-003, Brasil.

jcosta@epidemio-ufpel.org.br

\section{Abstract}

The aim of this study was to evaluate the prevalence of physical inactivity and associated factors in adult women (20-60 years) in São Leopoldo, Rio Grande do Sul, Brazil. A cross-sectional population-based study was carried out in 40 census tracts of the city. Physical inactivity was defined as no leisure-time physical activity or light-intensity activities no more than once a week. Of the 1,026 women interviewed, 380 (37.0\%; 95\% CI: 34.0-40.0) were classified as inactive. Another 609 (59.3\%) reported moderate or vigorous activities less than three times a week. In the crude analysis, physical inactivity was higher among non-white women, those with higher parity, with less schooling, low income, and obese. After adjustment, only the effect of body mass index remained statistically significant. Local and national policies for counseling and environmental improvements are necessary to reverse the high prevalence of physical inactivity observed in the community.

Motor Activity; Leisure Activities; Physical Fitness; Life Style; Women

\section{Introdução}

A chamada transição epidemiológica que ocorre no mundo tem se caracterizado pela modificação dos padrões de morbimortalidade, observando-se que as doenças crônicas não-transmissíveis vêm adquirindo magnitude e transcendência cada vez maiores 1,2 .

Entre os diversos fatores de risco associados à etiologia das doenças crônicas não-transmissíveis destaca-se o estilo de vida sedentário 3,4 . A literatura tem mostrado cada vez mais que a prática de atividade física é fator relevante na prevenção primária e como suporte terapêutico dessas doenças 5,6. A prática regular da atividade física aeróbica é a terapia de menor custo para promoção da saúde e prevenção de doenças 7,8. Seus benefícios são amplamente difundidos, tendo um importante papel nas prevenções primária e secundária da doença cardiovascular e no controle da pressão arterial 9,10. Também auxilia no controle das dislipidemias, na diminuição do nível de glicemia e no tratamento da obesidade 11,12 , na prevenção da osteoporose 13, do câncer de mama e cólon 14 .

$\mathrm{O}$ aumento da capacidade ventilatória $\left(\mathrm{VO}_{2}\right.$ max) decorrente da atividade física aeróbica regular traz consigo também uma maior disposição para todas as outras atividades cotidianas e menor cansaço ${ }^{15}$. Além disso, a atividade física age sobre o psique dos praticantes, diminuindo o isolamento, a depressão e favorecen- 
do a socialização e a formação de novos grupos, além de melhorar a auto-imagem 16.

Atualmente, campanhas de combate ao sedentarismo recomendam a prática de trinta minutos de atividades físicas na maioria dos dias da semana envolvendo os grandes grupos musculares, podendo ser feita de forma contínua ou fracionada 17. Manter alguma atividade física é melhor do que a inatividade. Porém, quando se recomenda prática fracionada ou leve, temse por objetivo quebrar a inércia para começar a adquirir hábitos de vida ativa e somente após algum tempo de prática regular, em ritmo submáximo (moderado) é que gradativamente vai se adquirindo os reais benefícios da prática da atividade física como fator de proteção para as doenças crônicas não-transmissíveis 12,18.

O conhecimento da realidade epidemiológica é um importante componente no planejamento de atividades de saúde pública ${ }^{19}$. Assim, o objetivo do presente estudo foi analisar as mulheres de 20 a 60 anos, residentes na zona urbana de São Leopoldo, Rio Grande do Sul, Brasil, quanto à prática de atividades físicas no lazer. Conhecendo-se as características das mulheres sedentárias ou insuficientemente ativas, é possível desencadear programas específicos de prevenção à saúde.

\section{Materiais e métodos}

Foi realizado um estudo transversal de base populacional com mulheres de 20 a 60 anos, residentes na zona urbana da cidade de São Leopoldo, Rio Grande do Sul. A cidade de São Leopoldo fica localizada ao sul do Brasil, na região do Vale do Rio dos Sinos, no Estado do Rio Grande do Sul. Faz parte da grande Porto Alegre, situando-se a $32 \mathrm{~km}$ da capital gaúcha. De acordo com o Censo Demográfico de 2000 (Instituo Brasileiro de Geografia e Estatística. http: //www.ibge.gov.br, acessado em 15/Abr/2005), possuía 193.547 habitantes, sendo que deste total, 98.781 eram mulheres $(51,07 \%)$.

O presente estudo está dentro de um projeto de pesquisa sobre a saúde da mulher com múltiplos desfechos, realizado pela Universidade do Vale do Rio dos Sinos (UNISINOS), de março a dezembro de 2003.

Calculou-se um tamanho de amostra que permitisse identificar uma razão de prevalência de 2,0 para um nível de confiança de $95 \%$, poder estatístico de $80 \%$, mantida a razão de não expostos: expostos de 1:3 segundo distribuição de nível econômico.

Entre todos os desfechos estudados estimou-se a amostra a partir daquele que exigia o maior tamanho. Optou-se pelo tamanho de amostra que permitisse estudar diabetes mellitus: 1.086 pessoas. O tamanho da amostra foi aumentado em $10,0 \%$ devido a possíveis perdas, e em $15,0 \%$ para controlar fatores de confusão na análise dos dados, sendo necessário um total de 1.358 pessoas. A partir do número de pessoas necessário para a amostra (1.358), da média de pessoas por domicílio na cidade de São Leopoldo $(3,35)$ e conhecendo-se a proporcionalidade da população de mulheres na faixa etária de interesse $(28,2 \%)$, foi estabelecida a necessidade de serem visitados 1.437 domicílios ${ }^{20}$. A precisão e poder das análises apresentadas neste artigo estão mostrados na seção de discussão.

Foram sorteados 40 setores censitários entre os 270 existentes na zona urbana da cidade de São Leopoldo. Em cada conglomerado foi sorteado o quarteirão e a seguir, a esquina a partir da qual foi identificado o domicílio onde teve início a coleta de dados. Após o sorteio da primeira moradia a ser entrevistada, a seguinte não era visitada e a próxima entrava na seleção. Foram visitados 36 domicílios por setor.

Foi utilizado um questionário padronizado e pré-codificado, onde estava inserido o instrumento específico sobre hábitos de atividade física, adaptado do programa Agita Brasil 17. As questões sobre atividade física no lazer incluíam o tipo de atividade, o número de vezes praticada por semana e o nível de esforço percebido (leve, médio ou forte).

$\mathrm{Na}$ análise dos dados foram selecionadas duas variáveis dependentes. A primeira caracterizava as mulheres que eram sedentárias, ou seja, referiam não realizar atividade física no lazer ou informaram fazer alguma atividade uma vez por semana e de forma leve.

O outro desfecho analisou as características das mulheres insuficientemente ativas, ou seja, aquelas que informaram praticar atividade física no lazer de forma média ou forte, menos de três vezes por semana.

Todos os entrevistadores eram acadêmicos da UNISINOS. Receberam treinamento para aplicação dos questionários e padronização da coleta de medidas. Foi realizado estudo piloto em outro setor censitário não incluído na amostra. O estudo piloto teve como objetivo testar os instrumentos, treinar o pessoal na coleta de dados e na codificação. O controle de qualidade foi realizado aplicando-se questionários simplificados, aleatoriamente, a 10,0\% da amostra.

As variáveis demográficas incluídas no estudo foram: idade, cor da pele observada pelo entrevistador, estado civil (solteiras, casadas, em união, separadas, divorciadas ou viúvas) e 
número de filhos. Foram investigadas variáveis relacionadas à acumulação de bens materiais $\mathrm{e}$ anos de escolaridade para determinação do nível econômico segundo a Associação Nacional de Empresas de Pesquisa - ANEP (Critério de Classificação Econômica Brasil. http://www. anep.org.br, acessado em 13/Fev/2004). A classificação da ANEP é constituída por cinco categorias, sendo que o nível mais elevado é denominado como classe A. A outra variável sócioeconômica estudada foi renda familiar em salários mínimos.

Foram investigadas também as presenças de algumas doenças crônicas não-transmissíveis tais como: hipertensão arterial sistêmica, diabetes mellitus e obesidade. Definiu-se hipertensão arterial sistêmica por pressão arterial $\geq 140 / 90 \mathrm{mmHg}$ a partir da média de duas medidas (início e fim da aplicação do questionário individual), ou o uso atual de medicação anti-hipertensiva 21 . A medida de tensão arterial foi coletada com esfigmomanômetro aneróide, com o paciente apresentando membro superior direito despido. A presença de diabetes mellitus foi definida por meio de história de diagnóstico médico prévio, referido pela pessoa entrevistada. Foram aferidos peso (kg) e altura (m) para cálculo do índice de massa corporal (IMC, calculado como $\mathrm{kg} / \mathrm{m}^{2}$ ). As mulheres foram pesadas com o mínimo de vestes possível, em posição ereta, de costas para a escala, com afastamento lateral dos pés, em balança de banheiro com precisão de um quilograma, aferida semanalmente. A estatura foi coletada com as mulheres descalças, em posição ereta, com pés e calcanhares unidos, braços estendidos ao longo do corpo, em apnéia, usando-se antropômetro. Indivíduos com IMC variando entre 25,0 e $29,9 \mathrm{~kg} / \mathrm{m}^{2}$ foram considerados com sobrepeso e aqueles com IMC $\geq$ $30,0 \mathrm{~kg} / \mathrm{m}^{2}$ foram classificados como obesos 22 .

A entrada dos dados foi realizada usandose o programa Epi Info, em dupla entrada e posterior comparação, para se eliminar a probabilidade de erros de digitação. As análises univariada e bivariada dos dados foram realizadas utilizando-se os programas SPSS e Stata. Nessa fase, foram calculadas as razões de prevalência, os intervalos de confiança e testes estatísticos: qui-quadrado e tendência linear para as variáveis ordinais. O efeito do delineamento amostral para o desfecho "sedentarismo" entre as mulheres foi de 1,62 e, portanto, as análises levaram em consideração o desenho amostral, por meio do conjunto de comandos svy do stata. Para o desfecho "atividade física insuficiente", o efeito do delineamento foi de 1,04 e as análises não necessitaram de correção.
Para controle dos fatores de confusão, a análise multivariada foi obtida por meio de regressão logística, sendo que as variáveis foram incluídas de acordo com modelo hierarquizado 23 . As variáveis demográficas e sócio-econômicas ficaram no primeiro nível, sendo as morbidades presentes no segundo nível. Vale ressaltar que as morbidades não são determinantes, e sim conseqüências do sedentarismo. Sendo assim, estas foram incluídas no último nível de análise. $O$ valor-p para manutenção das variáveis no modelo foi de 0,20. Manteve-se o máximo de variáveis no modelo, na tentativa de se detectar fatores de confusão negativos. Apenas uma variável sócio-econômica foi mantida no modelo em virtude da possibilidade de colinearidade.

O projeto de pesquisa foi aprovado pelo Comitê de Ética em Pesquisa da Faculdade de Medicina, Universidade Federal de Pelotas, tendo como normas primordiais a liberdade de escolha de participação na pesquisa, o sigilo dos dados e a confiabilidade.

\section{Resultados}

O número total de mulheres entrevistadas foi de 1.026 , sendo detectadas $58(5,65 \%)$ perdas e recusas. A faixa etária com maior percentual de mulheres foi a dos 40 a 49 anos, correspondendo a $29,0 \%$. Verificou-se que aproximadamente $84,0 \%$ das mulheres eram de cor branca. Os dados mostraram que cerca da metade das mulheres entrevistadas eram casadas e tinham de um a dois filhos (Tabela 1).

Em relação às variáveis sócio-econômicas observou-se que mais de $65,0 \%$ das mulheres estavam inseridas classes B e C da classificação econômica da ANEP. Quanto à escolaridade, verificou-se que aproximadamente $44,0 \%$ tiveram no máximo sete anos de estudo. Os dados revelaram que $78,0 \%$ das mulheres tinham uma renda menor que três salários mínimos (Tabela 1).

Entre as morbidades investigadas foram encontradas as seguintes prevalências: $3,7 \%$ para diabetes mellitus, $22,7 \%$ para hipertensão arterial sistêmica e $47,7 \%$ para sobrepeso e obesidade (Tabela 1).

A primeira variável dependente analisada mostrou as características das mulheres classificadas como sedentárias. Entre as $1.026 \mathrm{mu}$ lheres entrevistadas, 380 (37,0\%; IC95\%: 34,040,0 ) foram classificadas como sedentárias.

Em relação às variáveis demográficas, verificou-se que as mulheres não-brancas tinham maior probabilidade de ser sedentárias. Os dados mostraram também que as solteiras refe- 
Tabela 1

Descrição da amostra quanto às variáveis sócio-econômicas, demográficas e de morbidade. São Leopoldo, Rio Grande do Sul, Brasil, 2003.

\begin{tabular}{|c|c|c|}
\hline Variável & n & $\%$ \\
\hline \multicolumn{3}{|l|}{ Idade (anos) } \\
\hline $20-29$ & 283 & 27,6 \\
\hline $30-39$ & 255 & 24,9 \\
\hline $40-49$ & 302 & 29,4 \\
\hline $50-60$ & 186 & 18,1 \\
\hline \multicolumn{3}{|l|}{ Cor da pele } \\
\hline Branca & 860 & 83,8 \\
\hline Não-branca & 166 & 16,2 \\
\hline \multicolumn{3}{|l|}{ Estado civil } \\
\hline Solteira & 220 & 21,4 \\
\hline Casada & 510 & 49,7 \\
\hline Em união & 149 & 14,5 \\
\hline Separada/divorciada/viúva & 147 & 14,3 \\
\hline \multicolumn{3}{|l|}{ Número de filhos } \\
\hline Nenhum & 223 & 21,7 \\
\hline $1-2$ & 497 & 48,4 \\
\hline $3-4$ & 241 & 23,5 \\
\hline 5 ou + & 65 & 6,3 \\
\hline \multicolumn{3}{|l|}{ Nível econômico } \\
\hline A & 87 & 8,5 \\
\hline B & 272 & 26,5 \\
\hline C & 402 & 39,2 \\
\hline$D$ e $E$ & 265 & 25,9 \\
\hline \multicolumn{3}{|l|}{ Escolaridade (anos) } \\
\hline 14 ou + & 145 & 14,1 \\
\hline $11-13$ & 257 & 25,0 \\
\hline $8-10$ & 169 & 16,5 \\
\hline $5-7$ & 247 & 24,1 \\
\hline $0-4$ & 208 & 20,3 \\
\hline \multicolumn{3}{|l|}{ Renda em salários mínimos } \\
\hline$>3$ & 214 & 20,8 \\
\hline $1-3$ & 427 & 41,6 \\
\hline$<1$ & 373 & 36,4 \\
\hline \multicolumn{3}{|l|}{ Diabetes referido pelo médico } \\
\hline Não & 988 & 96,3 \\
\hline $\operatorname{Sim}$ & 38 & 3,7 \\
\hline \multicolumn{3}{|l|}{ Hipertensão arterial sistêmica } \\
\hline Não & 793 & 77,3 \\
\hline $\operatorname{Sim}$ & 233 & 22,7 \\
\hline \multicolumn{3}{|l|}{ Índice de massa corporal } \\
\hline Normal & 536 & 52,2 \\
\hline Sobrepeso & 311 & 30,3 \\
\hline Obesidade & 179 & 17,4 \\
\hline
\end{tabular}

riam praticar mais atividade física que aquelas classificadas nas outras categorias. Quanto ao número de filhos foram observadas diferenças estatisticamente significativas. À proporção que aumentava o número de filhos, elevava-se o percentual de mulheres que referiam sedentarismo. Não foram encontradas diferenças estatisticamente significativas quanto à idade (Tabela 2).

Em relação ao nível econômico, constatouse que as mulheres inseridas nas classes D e E referiram menor prática de atividade física do que as inseridas na classe A, contudo, o limite inferior do intervalo de confiança foi abaixo da unidade (Tabela 2).

Verificou-se que as mulheres com menor escolaridade apresentavam maior prevalência de sedentarismo. As mulheres com até quatro anos de estudo apresentavam probabilidade $60,0 \%$ maior de sedentarismo quando comparadas com aquelas de maior escolaridade. $\mathrm{Ob}$ servou-se significância estatística no teste de tendência linear, ou seja, à proporção que diminuía a escolaridade aumentava a prevalência de sedentarismo (Tabela 2 ).

Foram observadas diferenças estatisticamente significativas em relação à renda familiar, porém não houve uma tendência linear clara, sendo o grupo intermediário o que apresentou menor prevalência de sedentarismo. Além disso, os limites inferiores dos intervalos de confiança foram menores do que a unidade (Tabela 2).

Observou-se que as mulheres obesas apresentavam uma probabilidade $41,0 \%$ maior de serem sedentárias em relação às com IMC normal (Tabela 2).

Nesta análise não foram encontradas diferenças estatisticamente significativas quanto à presença de diabetes mellitus e hipertensão arterial sistêmica (Tabela 2).

Em relação à prática de atividade física no lazer três ou mais vezes por semana, com intensidade referida de média a forte, somente 37 (3,6\%; IC95\%: 2,5-4,7) foram classificadas como ativas. A apresentação dos resultados neste desfecho foi realizada demonstrando-se as características das mulheres insuficientemente ativas, ou seja, aquelas que não se enquadravam nessa definição.

A análise revelou diferenças estatisticamente significativas apenas na variável de classificação econômica da ANEP. Verificou-se que as mulheres das classes C, D e E apresentavam quase $10,0 \%$ a mais de probabilidade de serem classificadas como insuficientemente ativas quando comparadas com as da classe A. O teste de tendência linear foi estatisticamente sig- 
Tabela 2

Análise de sedentarismo em relação às variáveis sócio-econômicas, demográficas e de algumas morbidades. São Leopoldo, Rio Grande do Sul, Brasil, 2003.

\begin{tabular}{|c|c|c|c|c|c|}
\hline \multirow[t]{2}{*}{ Variável } & \multicolumn{2}{|c|}{ Sedentárias } & \multirow{2}{*}{$\begin{array}{l}\text { Razão de } \\
\text { prevalência }\end{array}$} & \multirow[t]{2}{*}{$1 \mathrm{IC} 95 \%$} & \multirow[t]{2}{*}{ valor $p$} \\
\hline & $\mathrm{n}$ & $\%$ & & & \\
\hline Idade (anos) & & & & & 0,71 \\
\hline $20-29$ & 97 & 34,3 & 1,00 & & \\
\hline $30-39$ & 95 & 37,3 & 1,08 & $0,83-1,41$ & \\
\hline $40-49$ & 118 & 39,1 & 1,14 & $0,89-1,45$ & \\
\hline $50-60$ & 70 & 37,6 & 1,10 & $0,83-1,45$ & \\
\hline Cor da pele & & & & & 0,02 \\
\hline Branca & 304 & 35,3 & 1,00 & & \\
\hline Não-branca & 76 & 45,8 & 1,30 & $1,05-1,60$ & \\
\hline Estado Civil & & & & & 0,03 \\
\hline Solteira & 60 & 27,3 & 1,00 & & \\
\hline Casada & 200 & 39,2 & 1,46 & $1,13-1,88$ & \\
\hline Em união & 57 & 38,3 & 1,42 & $1,00-2,02$ & \\
\hline Separada/divorciada/viúva & 63 & 42,9 & 1,59 & $1,13-2,24$ & \\
\hline Número de filhos* & & & & & 0,006 \\
\hline Nenhum & 58 & 26,0 & 1,00 & & \\
\hline $1-2$ & 193 & 38,8 & 1,49 & $1,21-1,84$ & \\
\hline $3-4$ & 101 & 41,9 & 1,61 & $1,30-2,00$ & \\
\hline 5 ou + & 28 & 43,1 & 1,66 & $1,22-2,24$ & \\
\hline Nível econômico & & & & & 0,24 \\
\hline$A$ & 23 & 26,4 & 1,00 & & \\
\hline B & 101 & 37,1 & 1,40 & $0,93-2,13$ & \\
\hline C & 145 & 36,1 & 1,36 & $0,88-2,12$ & \\
\hline$D$ e $E$ & 111 & 41,9 & 1,58 & $0,98-2,54$ & \\
\hline Escolaridade (anos)* & & & & & 0,04 \\
\hline 14 ou + & 39 & 26,9 & 1,00 & & \\
\hline $11-13$ & 85 & 33,1 & 1,23 & $0,83-1,81$ & \\
\hline $8-10$ & 68 & 40,2 & 1,50 & $1,03-2,18$ & \\
\hline $5-7$ & 98 & 39,7 & 1,48 & $1,00-2,17$ & \\
\hline $0-4$ & 90 & 43,3 & 1,60 & $1,09-2,33$ & \\
\hline Renda em salários mínimos & & & & & 0,02 \\
\hline$>3$ & 77 & 36,0 & 1,00 & & \\
\hline $1-3$ & 144 & 33,7 & 0,94 & $0,72-1,22$ & \\
\hline$<1$ & 157 & 42,1 & 1,17 & $0,89-1,53$ & \\
\hline Diabetes mellitus referido pelo médico & & & & & 0,34 \\
\hline Não & 369 & 37,3 & 1,00 & & \\
\hline Sim & 11 & 28,9 & 0,78 & $0,46-1,32$ & \\
\hline Hipertensão arterial sistêmica & & & & & 0,68 \\
\hline Não & 291 & 36,7 & 1,00 & & \\
\hline Sim & 89 & 38,2 & 1,03 & $0,88-1,22$ & \\
\hline Índice de massa corporal ${ }^{*}$ & & & & & 0,005 \\
\hline Normal & 180 & 33,6 & 1,00 & & \\
\hline Sobrepeso & 115 & 37,0 & 1,10 & $0,90-1,33$ & \\
\hline Obesidade & 85 & 47,5 & 1,41 & $1,16-1,72$ & \\
\hline
\end{tabular}

IC95\% = intervalo de confiança de 95\%.

$*<0,05$ para tendência linear. 
nificativo, revelando que a cada mudança de categoria aumentava a razão de prevalência (Tabela 3).

Foram encontrados também testes de tendência linear com significância estatística nas variáveis idade e escolaridade. Ou seja, à proporção que aumentava a idade, aumentavam as probabilidades das mulheres serem insuficientemente ativas. Por outro lado, na mesma proporção que diminuía a escolaridade, aumentava a prevalência das mulheres insuficientemente ativas (Tabela 3 ).

Para controle dos fatores de confusão na análise das mulheres classificadas como sedentárias foi realizada regressão logística com ingresso das variáveis seguindo modelo hierarquizado.

No ajuste do primeiro nível, as variáveis cor da pele, estado civil, número de filhos e escolaridade perderam significância estatística. No entanto, as tendências foram mantidas e algumas categorias apresentaram intervalos de confiança com limite inferior acima da unidade (Tabela 4). O efeito observado de que as mulheres obesas tinham maior probabilidade de sedentarismo foi preservado (Tabela 4).

\section{Discussão}

O objetivo do presente estudo era caracterizar as mulheres quanto à sua prática de atividade física no lazer, de forma a subsidiar os gestores de saúde com o intuito de se estimular a promoção da saúde e prevenção de doenças. Os resultados deste estudo podem ser norteadores do planejamento e implementação de políticas, projetos e programas visando ao aumento da prática da atividade física pela população, como tentativa de reverter o elevado percentual de sedentarismo.

Os dados do presente estudo demonstraram que $37,0 \%$ das mulheres de São Leopoldo eram sedentárias, 59,4\% insuficientemente ativas e somente 3,6\% ativas. Assim, 96,4\% das mulheres não estavam se beneficiando da maior parte da proteção à saúde fornecida pela prática de atividades físicas.

A maioria dos estudos sobre a prática de atividades físicas existente foi realizada com homens ou com a população em geral, sem diferenciar por sexo. Sendo que, os poucos estudos que diferenciavam a atividade física no lazer por sexo demonstravam que a prevalência de sedentarismo era maior em mulheres do que em homens 5. Quando todos os domínios da atividade física são avaliados (atividades ocupacionais, deslocamentos, de lazer e serviços domésticos), as diferenças por sexo tendem a desaparecer 24,25.

A comparação das prevalências entre diversos estudos é um grande problema na área da atividade física. A utilização de instrumentos e pontos de corte diferentes torna os estudos pouco comparáveis. Estudo realizado na cidade de São Paulo 26 encontrou uma prevalência de sedentarismo de 69,3\%. Dois estudos nacionais que avaliaram as quatro esferas da atividade física encontraram prevalência de inatividade em torno de 40,0-45,0\% 24,25.

No Município do Rio de Janeiro, 4.331 indivíduos acima de 12 anos foram incluídos em inquérito domiciliar. As mulheres realizavam menos atividades físicas no lazer do que os homens, sendo que a prevalência encontrada entre as mulheres foi de $77,8 \% 27$. Em outro estudo realizado numa universidade no Estado do Rio de Janeiro, que incluiu 4.030 funcionários de 20 a 60 anos, foi observado que as mulheres acumularam significativamente menos energia na prática de atividades físicas no lazer do que os homens 28.

No Estado do Rio Grande do Sul, estudo feito nos municípios sede de 19 coordenadorias regionais de saúde, com uma amostra de 1.066 participantes acima de 19 anos, mostrou que a prevalência de sedentarismo era de $71,3 \%$, sendo $71,4 \%$ no sexo masculino e $71,3 \%$ no sexo feminino, portanto, sem diferença por sexo 29.

No Canadá, em 1995, 62,0\% dos entrevistados não eram suficientemente ativos para colher os benefícios de saúde de um estilo de vida fisicamente ativo, sendo que o percentual encontrado para as mulheres foi de $67,0 \%$. O estudo revelou que somente $34,0 \%$ dos canadenses entre 25 e 55 anos eram fisicamente ativos, indicando também o sedentarismo como a principal causa das doenças crônicas não-transmissíveis e de morte prematura 30 . Nos Estados Unidos, $24,0 \%$ dos adultos eram totalmente sedentários, 54,0\% eram insuficientemente ativos e apenas $22,0 \%$ dos adultos praticavam no tempo livre atividades físicas no nível recomendado 31 .

Quando se avalia a associação entre atividade física e doenças crônicas, o fenômeno da causalidade reversa deve ser lembrado. Indivíduos diagnosticados e com informações sobre a enfermidade podem procurar mais fatores de proteção ${ }^{32}$. Isso se aplica para as variáveis: hipertensão arterial, diabetes mellitus e IMC.

A principal limitação do presente estudo foi não estabelecer a duração de tempo total por sessão das atividades físicas no lazer, uma vez que tradicionalmente se classificam como ativos os indivíduos que realizam trinta minutos 
Tabela 3

Características das mulheres insuficientemente ativas em relação às variáveis sócio-econômicas, demográficas e de algumas morbidades. São Leopoldo, Rio Grande do Sul, Brasil, 2003.

\begin{tabular}{|c|c|c|c|c|c|}
\hline \multirow[t]{2}{*}{ Variável } & \multicolumn{2}{|c|}{ Insuficientemente ativas } & \multirow{2}{*}{$\begin{array}{l}\text { Razão de } \\
\text { prevalência }\end{array}$} & \multirow[t]{2}{*}{ IC95\% } & \multirow[t]{2}{*}{ valor $p$} \\
\hline & $\mathrm{n}$ & $\%$ & & & \\
\hline Idade (anos)* & & & & & 0,08 \\
\hline $20-29$ & 268 & 94,7 & 1,00 & & \\
\hline $30-39$ & 243 & 95,3 & 1,01 & $0,97-1,05$ & \\
\hline $40-49$ & 296 & 98,0 & 1,03 & $1,00-1,07$ & \\
\hline $50-60$ & 182 & 97,8 & 1,03 & $1,00-1,07$ & \\
\hline Cor da pele & & & & & 0,64 \\
\hline Branca & 830 & 96,5 & 1,00 & & \\
\hline Não-branca & 159 & 95,8 & 0,99 & $0,96-1,03$ & \\
\hline Estado civil & & & & & 0,27 \\
\hline Solteira & 212 & 96,4 & 1,00 & & \\
\hline Casada & 490 & 96,1 & 1,00 & $0,97-1,03$ & \\
\hline Em união & 141 & 94,6 & 0,98 & $0,94-1,03$ & \\
\hline Separada/divorciada/viúva & 146 & 99,3 & 1,03 & $1,00-1,06$ & \\
\hline Número de filhos & & & & & 0,30 \\
\hline Nenhum & 212 & 95,1 & 1,00 & & \\
\hline $1-2$ & 479 & 96,4 & 1,01 & $0,98-1,05$ & \\
\hline $3-4$ & 298 & 97,4 & 1,02 & $0,99-1,06$ & \\
\hline Nível econômico** & & & & & 0,003 \\
\hline A & 78 & 89,7 & & & \\
\hline B & 261 & 96,0 & 1,07 & $0,99-1,15$ & \\
\hline C & 390 & 97,0 & 1,08 & $1,01-1,16$ & \\
\hline E & 260 & 98,1 & 1,09 & $1,02-1,18$ & \\
\hline Escolaridade (anos)* & & & & & 0,19 \\
\hline 14 ou + & 136 & 93,8 & 1,00 & & \\
\hline $11-13$ & 247 & 96,1 & 1,05 & $1,00-1,09$ & \\
\hline $8-10$ & 161 & 95,3 & 0,98 & $0,99-1,09$ & \\
\hline $5-7$ & 241 & 97,6 & 0,97 & $0,96-1,07$ & \\
\hline $0-4$ & 204 & 98,1 & 0,99 & $0,98-1,08$ & \\
\hline Renda em salários mínimos & & & & & 0,47 \\
\hline$>3$ & 142 & 95,3 & 1,00 & & \\
\hline $1-3$ & 170 & 95,5 & 1,00 & $0,96-1,05$ & \\
\hline$<1$ & 666 & 96,9 & 1,02 & $0,98-1,06$ & \\
\hline Diabetes mellitus referido pelo médico & & & & & 0,60 \\
\hline Não & 952 & 96,4 & 1,00 & & \\
\hline $\operatorname{Sim}$ & 37 & 97,4 & 1,01 & $0,96-1,07$ & \\
\hline Hipertensão arterial sistêmica & & & & & 0,82 \\
\hline Não & 765 & 96,5 & 1,00 & & \\
\hline Sim & 224 & 96,1 & 1,00 & $0,97-1,03$ & \\
\hline Índice de massa corporal & & & & & 0,16 \\
\hline Normal & 511 & 95,3 & 1,00 & & \\
\hline Sobrepeso & 304 & 97,7 & 1,03 & $1,00-1,05$ & \\
\hline Obesidade & 174 & 97,2 & 1,02 & $0,99-1,05$ & \\
\hline
\end{tabular}

IC95\% = intervalo de confiança de 95\%.

* $<0,05$ para tendência linear;

** 0,001 para tendência linear. 
Tabela 4

Análise multivariada dos fatores sócio-econômicos, demográficos e de morbidades com sedentarismo. São Leopoldo, Rio Grande do Sul, Brasil, 2003.

\begin{tabular}{|c|c|c|c|}
\hline Variável & $\begin{array}{c}\text { Razão de } \\
\text { prevalência }\end{array}$ & IC95\% & valor $\mathrm{p}$ \\
\hline Idade (anos) & & & 0,74 \\
\hline $20-29$ & 1,00 & & \\
\hline $30-39$ & 0,89 & $0,57-1,40$ & \\
\hline $40-49$ & 0,88 & $0,58-1,34$ & \\
\hline $50-60$ & 0,79 & $0,50-1,24$ & \\
\hline Cor da pele & & & 0,08 \\
\hline Branca & 1,00 & & \\
\hline Não-branca & 1,40 & $0,96-2,03$ & \\
\hline Estado civil & & & 0,13 \\
\hline Solteira & 1,00 & & \\
\hline Casada & 1,49 & $1,00-2,23$ & \\
\hline Em união & 1,30 & $0,72-2,33$ & \\
\hline Separada/divorciada/viúva & 1,72 & $1,06-2,79$ & \\
\hline Número de filhos & & & 0,09 \\
\hline Nenhum & 1,00 & & \\
\hline $1-2$ & 1,43 & $1,05-1,94$ & \\
\hline $3-4$ & 1,50 & $1,02-2,20$ & \\
\hline 5 ou + & 1,51 & $0,87-2,63$ & \\
\hline Escolaridade (anos) & & & 0,41 \\
\hline 14 ou + & 1,00 & & \\
\hline $11-13$ & 1,32 & $0,80-2,19$ & \\
\hline $8-10$ & 1,59 & $0,95-2,65$ & \\
\hline $5-7$ & 1,45 & $0,85-2,49$ & \\
\hline $0-4$ & 1,64 & $0,91-2,97$ & \\
\hline Diabetes mellitus referido pelo médico* & & & 0,15 \\
\hline Não & 1,00 & & \\
\hline $\operatorname{Sim}$ & 0,58 & $0,28-1,22$ & \\
\hline Hipertensão arterial sistêmica* & & & 0,22 \\
\hline Não & 1,00 & & \\
\hline $\operatorname{Sim}$ & 0,82 & $0,60-1,13$ & \\
\hline Índice de massa corporal* & & & 0,007 \\
\hline Normal & 1,00 & & \\
\hline Sobrepeso & 1,10 & $0,80-1,50$ & \\
\hline Obesidade & 1,83 & $1,23-2,72$ & \\
\hline
\end{tabular}

IC95\% = intervalo de confiança de 95\%.

* Resultados ajustados para idade, cor da pele, estado civil, número de filhos e escolaridade. de atividade física na maioria dos dias da semana, em intensidade moderada 33,34. Como forma de atenuar os problemas de classificação cometidos no instrumento de pesquisa, deve-se ressaltar que as informações coletadas sobre as sessões de atividade física foram sempre superiores a dez minutos.

Outra limitação do estudo foi a coleta de informações referentes exclusivamente à prática de atividades físicas no lazer, não sendo mensurados o esforço físico no trabalho, deslocamentos e as atividades domésticas, os quais poderiam aumentar a prevalência de indivíduos classificados como suficientemente ativos, conforme sugerido recentemente 25 . Como o projeto envolvia diversos desfechos e exposições, por motivos logísticos, optou-se por investigar apenas a atividade física no lazer.

Dentre as vantagens, o estudo foi de base populacional envolvendo a população feminina residente na zona urbana de São Leopoldo. A distribuição percentual por faixa etária da amostra foi semelhante à estimativa apresentada no Censo Demográfico 2000, concluindose que a amostra foi representativa da zona urbana de São Leopoldo.

Além disso, o cálculo da amostra do estudo como um todo foi estimado a partir do desfecho que exigia o maior número de indivíduos. Assim, ao se utilizar a prevalência de sedentarismo, a margem de erro da estimativa foi de apenas três pontos percentuais.

Dessa forma, para reverter o quadro encontrado, sugere-se a implementação de políticas municipais, estaduais e nacionais de prevenção e combate ao sedentarismo, com ênfase na divulgação de seus benefícios e na criação de aparelhos e espaços públicos para prática de atividade física na comunidade. Aconselha-se também a oferta de cursos de atualização a todos os profissionais de saúde, esclarecendo sobre a importância da atividade física e a necessidade de recomendá-la, tendo-se o cuidado de implantar este procedimento na maioria dos serviços de saúde.

Sugere-se ainda, para viabilização da criação de tais programas, parcerias com outras instituições tais como secretarias de educação, clubes esportivos e associações comunitárias, com o objetivo de mudanças de hábitos na população, ensinando desde cedo nas escolas a importância da atividade física para saúde. 


\section{Resumo}

O objetivo desta pesquisa foi identificar a prevalência de sedentarismo e fatores associados em mulheres adultas (20-60 anos) de São Leopoldo, Rio Grande do Sul, Brasil. Foi realizado um estudo transversal de base populacional em quarenta setores censitários da cidade. Sedentarismo foi definido como nenhuma atividade física no lazer ou apenas uma vez por semana, de intensidade leve. Dentre as 1.026 mulheres entrevistadas, 380 (37,0\%; IC95\%: 34,0-40,0) foram classificadas como sedentárias. Além destas, 609 (59,3\%) relataram praticar atividades físicas moderadas ou vigorosas no lazer menos de três vezes por semana. $\mathrm{Na}$ análise bruta o sedentarismo foi mais comum entre mulheres não-brancas, com maior número de filhos, de menor escolaridade, de menor renda e obesas. $\mathrm{Na}$ análise ajustada apenas o efeito do índice de massa corporal manteve-se estatisticamente significativo. A implementação de políticas municipais, estaduais e nacionais com ênfase em aconselhamento e melhorias ambientais é necessária para reversão da alta prevalência de sedentarismo observada na comunidade.

Atividade Motora; Atividades de Lazer; Aptidão Física; Estilo de Vida; Mulheres

\section{Colaboradores}

C. R. Masson, J. S. Dias-da-Costa, P. C. Hallal participaram da coleta de dados, revisão bibliográfica, análise e discussão do artigo. M. T. A. Olinto, S. Meneghel, C. C. Costa, F. Bairros colaboraram na coleta de dados, análise e discussão do artigo. Todos os autores contribuíram para a elaboração do artigo final.

\section{Referências}

1. Franco LJ. Epidemiologia do diabetes mellitus. In: Lessa I, organizador. $\mathrm{O}$ adulto brasileiro e as doenças da modernidade. Epidemiologia das doenças crônicas não-transmissíveis. São Paulo: Editora Hucitec/Rio de Janeiro: ABRASCO; 1998. p. 123-37.

2. Almeida MB, Araújo CGS. Effects of aerobic training on heart rate. Rev Bras Med Esporte 2003; 9:113-20.

3. Ho KK, Pinsky JL, Kannel WB, Levy D. The epidemiology of heart failure: the Framingham Study. J Am Coll Cardiol 1993; 22 (4 Suppl A):6A-13A.

4. Castro MC. El uso de la actividad física como prevención en salud. Lecturas: Educación Física y Deportes, Revista Digital 2001; 41. http:/ / www. efdeportes.com/efd41/altern.htm (acessado em 18/Abr/2004).

5. Blair SN, Kampert JB, Kohl HW 3rd, Barlow CE, Macera CA, Paffenbarger Jr. RS, et al. Influences of cardiorespiratory fitness and other precursors on cardiovascular disease and all-cause mortality in men and women. JAMA 1996; 276:205-10.

6. Haennel RG, Lemire F. Physical activity to prevent cardiovascular disease. Can Fam Physician 2002; 48:65-71.

7. Bowne DW, Russell ML, Morgan JL, Optenberg SA, Clarke AE. Reduced disability and health care costs in an industrial fitness program. J Occup Med 1984; 26:809-16.

8. Woolhandler S, Campbell T, Himmelstein DU. Costs of health care administration in the United States and Canada. N Engl J Med 2003; 349:76875.

9. Paffenbarger Jr. RS, Hyde RT, Wing AL, Lee IM, Jung DL, Kampert JB. The association of changes in physical-activity level and other lifestyle characteristics with mortality among men. N Engl J Med 1993; 328:538-45.

10. Elrick H. Exercise - the best prescription. Phys Sportsmed 1996; 24(2). http://www.physsports med.com/issues/1996/02_96/elrickpa.htm (acessado em 15/Ago/2004).

11. Blair SN. Evidence for success of exercise in weight loss and control. Ann Intern Med 1993; 119 (7 Pt 2):702-6.

12. Fletcher GF, Balady G, Blair SN, Blumenthal J, Caspersen C, Chaitman B, et al. Statement on exercise: benefits and recommendations for physical activity programs for all Americans. A statement for health professionals by the Committee on Exercise and Cardiac Rehabilitation of the Council on Clinical Cardiology, American Heart Association. Circulation 1996; 94:857-62.

13. Carvalho T, Nóbrega ACL, Lazzoli JK, Magni JRT, Rezende LR, Drummond FA, et al. Posição oficial da Sociedade Brasileira de Medicina do Esporte: atividade física e saúde. Rev Bras Med Esporte 1996; 2:79-81.

14. Battaglini C, Battaglini B, Bottaro M. The effects of physical exercise on cancer: a review. Lecturas: Educación Física y Deportes, Revista Digital 2003; 57. http://www.efdeportes.com/efd57/cancer. htm (acessado em 23/Set/2003).

15. Fabre C, Traisnel C, Mucci P. Benefits of gymnas- 
tic activity on fitness, cognitive function and medication in elderly women. Sci Sports 2003; 18:196201.

16. Franklin B, Bonzheim K, Warren J, Haapaniemi S, Byl N, Gordon N. Effects of a contemporary, exercise-based rehabilitation and cardiovascular riskreduction program on coronary patients with abnormal baseline risk factors. Chest 2002; 122:33843.

17. Ministério da Saúde. Agita Brasil: guia para agentes multiplicadores. Brasília: Ministério da Saúde; 2001.

18. Ministério da Saúde. Programa de educação e saúde através do exercício físico e do esporte. Brasília: Ministério da Saúde; 2003.

19. Beaglehole R, Bonita R, Kjellström T. Epidemiologia básica. São Paulo: Editora Santos; 2001.

20. Barros FC, Victora CG, Vaughan JP. Epidemiologia da saúde infantil: um manual para diagnósticos comunitários. São Paulo: Editora Hucitec/Fundo das Nações Unidas para a Infância; 1990.

21. World Health Organization. Hypertension and coronary heart disease: classification and criteria for epidemiological studies. Geneva: World Health Organization; 1959. (Technical Report Series n. 168).

22. World Health Organization. Obesity: preventing and managing the global epidemic. Geneva: World Health Organization; 1998.

23. Victora CG, Huttly SR, Fuchs SC, Olinto MTA. The role of conceptual frameworks in epidemiological analysis: a hierarchical approach. Int J Epidemiol 1997; 26:224-47.

24. Matsudo SM, Matsudo VR, Araújo T, Andrade D, Andrade E, Oliveira L, et al. Nível de atividade física na população do Estado de São Paulo: análise de acordo com o gênero, idade, nível sócioeconômico, distribuição geográfica de reconhecimento. Rev Bras Ciênc Mov 2002; 10:41-50.

25. Hallal PC, Victora CG, Wells JCK, Lima RC. Physical inactivity: prevalence and associated variables in Brazilian adults. Med Sci Sports Exerc 2003; 35:1894-900.
26. Rego RA, Berardo FAN, Rodrigues SSR, Oliveira ZMA, Oliveira MB, Vasconcellos C, et al. Fatores de risco para doenças crônicas não-transmissíveis: inquérito domiciliar no Município de São Paulo, SP (Brasil). Metodologia e resultados preliminares. Rev Saúde Pública 1990; 24:277-85.

27. Gomes VB, Siqueira KS, Sichieri R. Atividade física em uma amostra probabilística do Município do Rio de Janeiro. Cad Saúde Pública 2001; 17: 969-76.

28. Salles-Costa R, Werneck GL, Lopes CS, Faerstein E. Associação entre fatores sócio-demográficos e prática de atividade física de lazer no Estudo PróSaúde. Cad Saúde Pública 2003; 19:1095-105.

29. Secretaria de Saúde do Rio Grande do Sul. Pesquisa sobre fatores de risco para a doença coronariana no Rio Grande do Sul. Boletim Epidemiológico da Secretaria de Saúde do Rio Grande do Sul 2001; 3:4-5.

30. Katzmarzyk PT, Gledhill N, Shephard RJ. The economic burden of physical inactivity in Canada. CMAJ 2000; 163:1435-40.

31. Centers for Disease Control and Prevention/ American College of Sports Medicine. Physical activity and public health. JAMA 1995; 273:402-7.

32. Andrade ALSS, Zicker F. Estudos de prevalência. In: Andrade ALSS, Zicker F, organizadores. Métodos de investigação epidemiológica em doenças transmissíveis. v. I. Brasília: Organização PanAmericana da Saúde/Fundação Nacional de Saúde; 1997. p. 33-42.

33. Carvalho DCL, Rosim GC, Gama LOR, Tavares MR, Tribioli RA, Santos IR, et al. Tratamentos não farmacológicos na estimulação da osteogênese. Rev Saúde Pública 2002; 36:647-54.

34. Leitão MB, Lazzoli JK, Oliveira MAB, Nóbrega ACL, Silveira GG, Carvalho T, et al. Posicionamento oficial da Sociedade Brasileira de Medicina do Esporte: atividade física e saúde na mulher. Rev Bras Med Esporte 2000; 6:215-20.

Recebido em 09/Nov/2004

Versão final reapresentada em 19/Abr/2005 Aprovado em 06/Mai/2005 